\title{
Erfahrungsbericht
}

\author{
Frauke Musial*
}

\section{Der „Leichtfuß“, die Berge, das Meer und ich - mit dem Rollstuhl in die Arktis}

https://doi.org/10.1515/spircare-2019-0007

Vorab online veröffentlicht 25. Juni 2019

Als ich gefragt wurde, über mein „Leben mit Beeinträchtigungen" zu schreiben, habe ich spontan und begeistert zugesagt! Wie toll, da interessiert sich jemand für das, was ich in den letzten Jahren erlebt habe, ich darf einmal nur über das schreiben, was mir wichtig ist, was mich beeindruckt, was mich bewegt. Nicht wissenschaftlich, ganz unkompliziert, herrlich! Und dann war es doch viel schwieriger, als ich es jemals erwartet hätte.

Wo soll ich anfangen? Vielleicht damit, dass ich chronisch krank bin. Bei mir wurde im Alter von 39 Jahren eine Multiple Sklerose diagnostiziert. Beschwerden hatte ich schon länger, ich war eigentlich sehr fit und sportlich, aber dann bekam ich beim Joggen Probleme. Ich bin mehrere Jahre von Arzt zu Arzt gelaufen, Neurologen, Neuroradiologen, Neurochirurgen, niemand hat mich ernstgenommen, keiner gründlich körperlich untersucht. Es ist bezeichnend, dass erst ein Psychiater kommen musste, der alles richtig gemacht hat, mich zum ersten Mal gründlich untersucht und letztlich die erforderliche Diagnostik angeordnet hat. Multiple Sklerose also - ich wollte erst einmal sterben. Ein langsamer Tod durch Dahinsiechen, nicht vorstellbar für mich.

Ich bin sicher durch all das gegangen, was andere $\mathrm{Pa}$ tienten auch kennen, Kampfgeist, Ignorieren, Leugnen, Durchhalten etc. Der Verlauf der Krankheit war deutlich schlechter als ursprünglich prognostiziert, es ging eigentlich rasend schnell im Verlauf von ca. 5 Jahren, bis ich am Stock, später an zwei Gehilfen ging und einen kleinen Scooter benutzte. Meine Gehstrecke wurde immer kürzer, Kortison half zwar im akuten Schub, aber ansonsten half nichts wirklich. Es ging einfach bergab.

Irgendwann schlug die Verzweiflung in Trotz um. Nur nicht vor der Krankheit kapitulieren, nicht aufgeben, ich lasse mir mein Leben doch nicht von einer idiotischen Krankheit stehlen. Viele Menschen haben mir dabei gehol-

*Korrespondenzautorin: Frauke Musial, UiT, The Arctic University of Norway - Community Medicine, Troms $\varnothing$ Norwegen,

E-Mail: frauke.musial@uit.no fen: Mein ehemaliger Chef, der mir vorschlug und erlaubte, einen Hund zu haben und mit zur Arbeit zu bringen („Da musst Du laufen, da kannst Du nicht zu Hause abhängen”), die damalige Schwerbehindertenbeauftragte, die selbst eine deutliche Gehbehinderung hatte („Sie sind Wissenschaftlerin? Da können Sie ja noch ewig arbeiten. Seien Sie froh, dass Sie kein Postbote sind"), und viele, liebe andere Menschen, mein Mann und meine Familie, Kollegen, Freunde, die mir nahestehen haben mich unterstützt. Ich war mit den Folgen meiner Krankheit niemals alleine, auch wenn man mit einer solchen Diagnose und Behinderung wohl im Prinzip immer alleine fertig werden muss. Es ging so, aber gut ging es mir nicht.

Heute, ca. 15 Jahre nach der Diagnose, geht es mir gut. Viel besser als in mancher Lebensphase, in der ich jung und gesund war und dachte, die Welt liegt mir zu Füßen und hat nur auf mich gewartet. In manchen Momenten durchflutet mich ein Glückgefühl, dass ich nicht in Worte fassen kann. Und selbst jetzt, wo ich diese Zeilen schreibe und an all das denke, was mich glücklich macht, muss ich mich ganz schön anstrengen, damit ich überhaupt weiter schreiben kann. Was ist geschehen?

So krank zu werden ist einfach Mist. Wenn man nur befristet angestellt ist und weiß, dass diese Diagnose bei jedem Menschen, der sie kennt, und damit auch bei Arbeitgebern, Angst auslöst, kann man sich vorstellen, was das bedeutet. Man kann versuchen, für eine Weile geheim zu halten, wie krank man wirklich ist, aber nicht mehr, wenn man de facto im Rollstuhl sitzt. Im Übrigen habe ich meine Erkrankung niemals verheimlicht, ich bin nun einmal ein Mensch mit MS, und das war ich auch, als man es noch nicht sehen konnte. Es wäre mir zu anstrengend gewesen, daraus ein Geheimnis machen zu wollen und ich habe damit überwiegend gute Erfahrungen gemacht. Im Jahr 2011 wäre mal wieder eine befristete Anstellung ausgelaufen und ich war wieder auf der Suche nach einem neuen Job. Dann wurde 2010 eine Stelle an einem Institut in Tromsø ausgeschrieben, ich habe mich beworben und wurde genommen. Meine erste, unbefristete Anstellung, in Troms $\varnothing$, in der Arktis, ca. 300 km nördlich vom Polarkreis.

Ich kannte die Arbeitsgruppe, ich war 2008 schon einmal in Troms $\varnothing$, besser auf einer Insel in der Nähe, der 


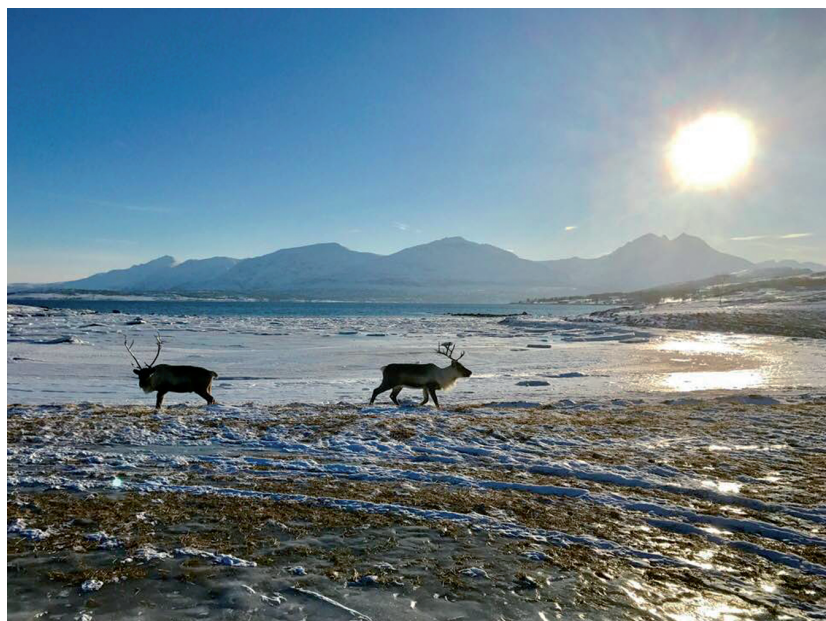

Foto: Agnete Egilsdatter Kristoffersen

„Sommerinsel“ Sommarøy gewesen, und war dort zu einem Workshop eingeladen. Es war großartig! Wissenschaftlich einfach unheimlich beeindruckend, und darüber hinaus richtig nette Leute. Es war schon Ende September und ganz schön kalt, und das Wetter so lala. Wenn ich mich heute daran erinnere, erinnere ich mich auch daran, dass ich viel geheult, oder zumindest mit den Tränen gekämpft habe. Warum? Ich weiß es eigentlich nicht so genau... ich bin nicht nahe am Wasser gebaut und ich war auch nicht traurig, es war einfach so wahnsinnig schön dort! Die Natur hat mich überwältigt, es raubt einem den Atem. Es gibt keine Worte, die wirklich beschreiben können, wie ergreifend die Landschaft dort ist. Es ist wild, es kann bedrohlich sein, hinter jeder Kurve auf unserer Fahrt von Tromsø nach Sommarøy ein neuer Eindruck. Sanfte Auen wechseln sich mit schroffen Felsen ab und überall ist das Meer. Mal ruhige und sanfte Buchten mit weißen Stränden wie Lagunen, mal eine peitschende Brandung an einer schroffen Steilküste und das alles innerhalb von fünf Minuten Fahrt. Ich werde es nie vergessen, ich saß auf der Terrasse des Arctic Hotels in Sommarøy und wusste, hier will ich hin.

Es ist das ehrfürchtige Staunen, der „sense of awe“, der mich bei meiner ersten Begegnung mit Nord-Norwegen überwältigt hat. Ich bin ein naturverbundener Mensch, ich war immer viel draußen, bin gewandert, ja, ich habe auch schon einmal einen Baum umarmt, wenn es mir schlecht ging. Meine Gehbehinderung hat mich zu großen Teilen der Möglichkeit beraubt, in der Natur zu sein. Hier kommt die Natur zu einem, man kann ihr quasi nicht entfliehen: sei es, wenn es im Sommer die ganze Nacht hell ist, oder wenn im Winter drei Tage Schneesturm herrscht, und niemand zur Arbeit fährt, weil es einfach zu gefährlich ist.

Ich habe meinen Dienst in Tromsø im März angetreten, also in einer Zeit, wo die Polarnacht schon vorbei ist,

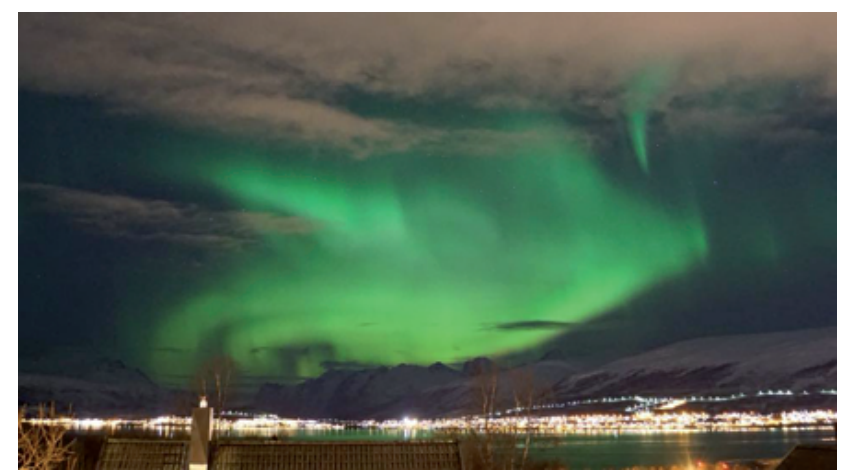

Foto: Agnete Egilsdatter Kristoffersen

aber die Tage noch kurz und die Nächte lang sind. Mein Weg zur Arbeit führt am Wasser vorbei, am Sandnessund mit Blick auf die Insel Kvaløya. Ich habe nicht gezählt, wie oft ich in den ersten Jahren morgens rechts heranfahren musste, überwältigt von dem Bild, das sich vor meinen Augen ausbreitete. Kvaløya, wie eine Christbaumkugel erleuchtet, davor der spiegelblanke Sund, in dem sich der Mond und die Sterne abbilden und darüber Nordlichter, die in unwirklichem, überirdischem Grün hin und her tanzen. Es tut beinahe weh, soviel Schönheit kann man fast nicht ertragen.

Man kann nicht Wale unter dem Nordlicht spielen und jagen sehen, ihr Prusten und Schnaufen hören, ohne das etwas mit einem geschieht. Man kann nicht in einer sternenklaren Nacht unter dem Nordlicht über ein Schneefeld durch eine Herde Rentiere reiten, ohne dass man sich verändert. Die Nacht ist ruhig, aber nicht still, das Wasser plätschert am Strand (kein Eis im Winter auf dem Meer, die „warme“ Nordatlantikströmung ...), die Pferde schnauben fröhlich vor sich hin, man hört das Knirschen ihrer Hufe im Schnee, man fühlt ihre Wärme, man bewegt sich schwerelos durch die verzauberte Nacht... .

Überhaupt, die Pferde, mein Pferd, Fràr, auf Deutsch: der „Leichtfuß“. Ein „Isländer“ (Islandpferd, eine aus Island stammende robuste Kleinpferderasse), was sonst hier oben, in dem rauen Klima. Eines der großartigsten Geschenke, die mir das Leben durch meine Freundin Agnete gemacht hat. Ich war früher in jungen Jahren eine gute Reiterin, habe dann aufgehört, und wollte immer wieder damit anfangen, bis ich krank wurde und der Plan einfach verschwand. Agnete hat mich wieder aufs' Pferd gesetzt („Papperlapapp, das Du nicht gehen kannst, heißt ja nicht, dass Du nicht reiten kannst“), und so war’s. Das ist jetzt die deutlich gekürzte und geschönte Version, ohne all die blauen Flecken und verzweifelten Momente, die es natürlich auch gab. Pferde sind meine Beine geworden, sie tragen mich überall dorthin, wo Pferd und ich uns hin trauen. Die Geschichte Nummer 2 meiner Reiterei, viele 


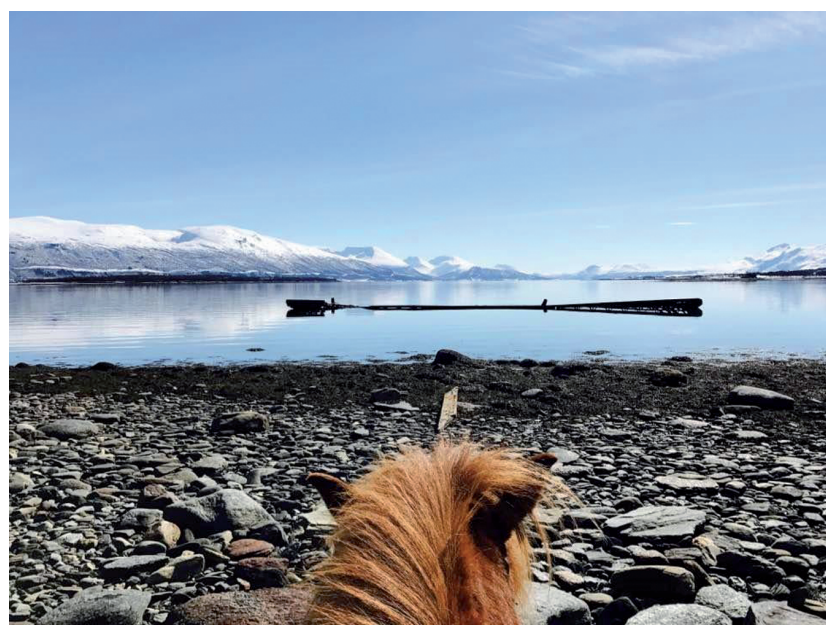

Foto: Agnete Egilsdatter Kristoffersen

Jahre später im Leben, ist also eine unglaubliche Geschichte von Freundschaft, Unterstützung, Gemeinschaft, und nicht zuletzt, Liebe.

So viele Menschen haben mir dabei geholfen, mich auf dem Pferd geführt, mich unterrichtet, auf mich aufgepasst, mich aufs Pferd geschoben, mich wieder aufgesammelt, Höhen und Tiefen geteilt. Die Geschichte meiner Reise in die Arktis ist auch eine Geschichte von Pferden und Menschen und von „Pferdemenschen”, Equestrians. Für mich ist es eindeutig der allerschönste, sekundäre Krankheitsgewinn, den es geben kann. Wer weiß, ob ich ohne diese Krankheit jemals im Leben wieder angefangen hätte zu reiten?

Wir haben Glück mit uns allen im Stall, wir sind definitiv kein Turnierstall, wir lieben die Natur, wir lieben es draußen zu sein, egal in welchem Wetter, und wir lieben Pferde. Wir haben dieselben Vorstellungen davon, wie man reiten und Pferde halten soll. Wir sind eine loyale, beinahe verschworene Gemeinschaft von Freunden. Aber was fasziniert uns so an Pferden? Pferde sind unglaublich klug, sie sind als Herdentiere - und Islandpferde wachsen besonders natürlich auf - besonders sozial und empfänglich für Emotionen. Emotionen übertragen sich unmittelbar zwischen Pferden und zwischen Mensch und Pferd. Es ist nicht möglich, ärgerlich oder gestresst zu reiten - das geht mit Sicherheit nicht gut. Damit sind Pferde eine ganz hervorragende „Achtsamkeitsübung”. Wenn ich mit einem fröhlichen, entspannten Pferd losreiten möchte, muss ich mich als fröhlicher entspannter Mensch auf dieses Pferd setzen.

An unserem kleinen Stall haben wir möglicherweise die höchste Wissenschaftlerdichte von allen Ställen im Umkreis und damit wahrscheinlich auch einen deutlich höheren Anteil an „Kopfmenschen” als andere Ställe. Aber alle berichten dasselbe, wenn sie am Stall sind und die

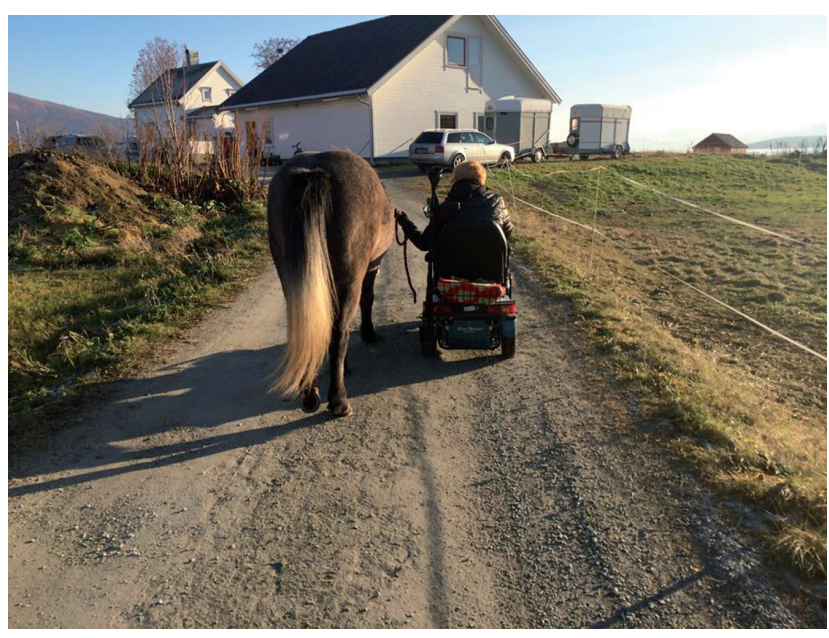

Foto: Ina Solberg Andreassen

Pferde hereinholen: Dann spielt nichts mehr eine Rolle, nur das Pferd. Pferde lehren uns, ganz präsent im Augenblick zu sein. Ich habe von Pferden mehr über mich selbst gelernt, als ich jemals über Pferde lernen kann. Ich kann mit meinem Körper kein Pferd mit Kraft oder Gewalt dazu bringen, irgendetwas für mich zu tun, wir müssen kommunizieren, wir müssen uns verstehen. Dann kommt noch das „Problem“ des somatosensorischen dazu: Man sitzt ja auf einem Pferd und hat eine direkte körperliche Verbindung. Das heißt: Alles, aber auch alles, was sich körperlich ausdrückt, wirkt und überträgt sich auf das Pferd, natürlich auch Gefühle. Pferde sind damit auch das perfekte „Gefühlsbiofeedback“. Das ist das große Geschenk, das mir die Pferde, allen voran mein Fràr, gemacht haben: $\mathrm{Zu}$ gang zu einem Teil von mir zu finden, den ich nicht wichtig genug genommen habe und dem ich zuvor nicht den Raum eingeräumt habe, den er verdient.

Es geht mir gut, danke! Die Krankheitsaktivität ist mehr oder weniger zum Stillstand gekommen. Wenn sich etwas getan hat, dann ist es besser geworden. Ich bin immer noch stark gehbehindert, aber die Progredienz ist weg. Vielleicht Zufall, eine Multiple Sklerose kann zum Stillstand kommen, auch wenn sie am Anfang sehr progredient war. Wer weiß schon, warum „meine” MS sich so verhält. Und jetzt kommt die große Plattitüde, die ich doch als so wahr und ehrlich empfinde: Ich möchte meine Krankheit nicht missen. Sicher, wenn ich jetzt wählen könnte, wieder gesund zu werden, würde ich natürlich gesund sein wollen. Aber vor die Wahl gestellt, mein Leben noch einmal ohne die letzten 15 Jahre mit MS zu leben, und dafür nicht hier in der Arktis zu sein und all das nicht erlebt (und gelernt) zu haben, was ich erleben durfte, würde ich mich dagegen entscheiden.

Ich habe Dinge gesehen und erleben dürfen, die ich ohne diese Erkrankung nie erlebt hätte. Ich bin mit Fràr 
unter dem Nordlicht über ein unberührtes Schneefeld galoppiert, mit freien Zügeln, die Arme seitlich ausgestreckt, völlig diesem wundervollen Moment ergeben. Wir haben die Mitternachtssonne vom Berggipfel aus gesehen, wie sie das Wasser beinahe berührt hat, und dann wieder am Horizont aufstieg; wir sind unter kreisenden Adlern durch das Watt gestiefelt, geblendet von der Sonne; wir sind durch den duftenden, feuchten Herbstwald gestreift, mit seinen leuchtenden Farben, und haben dem Plätschern der Wildbäche und dem „Singen“ der ziehenden Wildgänse gelauscht.
Ich bin kein sehr religiöser Mensch, doch hier ist die Natur meine Kathedrale. In dieser grandiosen Landschaft fühle ich eine Verbundenheit, die mich zutiefst bewegt und die mich verändert hat. Wenn ich das „Eine“ benennen sollte, was mein Herz hier so sehr berührt, dann ist es die Natur und das ehrfürchtige Staunen über ihre Schönheit, ihre Macht und Wildheit, und über ihre zeitliche Dimension, ihre Ewigkeit, in der meine Existenz nur ein Augenblick ist. 